\title{
L'appareil de Golgi : apport de la microscopie électronique tridimensionnelle à l'étude de sa structure et de ses fonctions
}

L'étude de l'appareil de Golgi en microscopie électronique stéréoscopique, utilisant des coupes épaisses et fines, permet de reconstituer le détail de sa structure. Comme l'avait indiqué Golgi lui-même il y a 90 ans, cet organite forme un réseau continu variant selon le type cellulaire. En microscopie électronique le ruban golgien est hétérogène, que ce soit dans l'axe longitudinal du ruban ou dans l'axe transversal cis-trans. Les protéines provenant du réticulum endoplasmique entrent dans l'appareil de Golgi sur sa face cis. Ces protéines sont glycosylées au cours de leur transit vers la face trans et quittent l'appareil de Golgi sous forme de glycoprotéines pour former des grains de sécrétion, des lysosomes, ou vont s'ajouter à la membrane cellulaire. La ségrégation des diverses glycoprotéines se fait sur la face trans du ruban golgien.

\section{Alain Rambourg Yves Clermont}

\section{ADRESSES}

A. Rambourg: professeur. Département de biologie du CEA, Saclay, France.

Y. Clermont : professeur. Département d'anatomie, université McGill, 3640 rue de l'Université, Montréal, Québec, H3A 2B2 Canada. n 1898, un médecin de l'université de Padoue, Camillo Golgi, prix Nobel de médecine en 1906, découvrit par hasard un nouvel organite intracellulaire. Alors qu'il étudiait la structure du tissu nerveux à l'aide de techniques d'imprégnation métallique mises au point dans son laboratoire, il observa dans le cytoplasme des cellules nerveuses la présence de filaments et de plaques intensément colorés par l'osmium [1]. En faisant varier la mise au point de son microscope, Golgi put se convaincre que ces structures, séparées les unes des autres lorsqu'il les examinait dans un seul plan de mise au point, formaient en réalité dans le cytoplasme un seul réseau continu auquel il donna le nom d'appareil réticulaire interne. Cependant, la variabilité des résultats obtenus par la méthode d'imprégnation prônée par Golgi et la difficulté d'observer une structure similaire dans la cellule vivante jetèrent le discrédit sur le nouvel organite : cinquante ans après sa découverte, l'appareil réticulaire interne ou appareil de Golgi était généralement considéré comme un artéfact.

Les premières observations réalisées en microscopie électronique confirmèrent l'existence, dans la plupart des cellules, d'une région correspondant à la structure décrite par Golgi, mais c'est à Dalton et Felix [2] que revint le mérite, en 1954, d'avoir convaincu la communauté scientifique de la réalité de l'appareil de Golgi. Examinant à l'aide du microscope électronique des coupes ultrafines imprégnées par des métaux lourds, ils démontrèrent la présence dans le cytoplasme d'un empilement caractéristique de citernes aplaties (les lamelles ou saccules) entouré de 
vésicules et de vacuoles. L'imprégnation par le tétroxyde d'osmium des saccules de ce complexe membranaire permettait d'établir une relation avec les observations originales de Golgi et de clore définitivement une discussion acharnée souvent fertile en épisodes pittoresques.

\section{Représentation classique de l'ultrastructure de l'appareil de Golgi}

Examiné sur des coupes ultrafines (environ 100 nanomètres d'épaisseur), par les méthodes conventionnelles de la microscopie électronique, l'appareil de Golgi semble constitué par un ensemble de piles de saccules séparées les unes des autres et distribuées au hasard dans le cytoplasme juxta- ou périnucléaire. Dans les cellules glandulaires, les piles de saccules occupent la région comprise entre le noyau et les grains de sécrétion, généralement groupés au pôle sécréteur de la cellule (figures 1 et 2 ). Chaque pile de saccules présente une polarité de structure qui a été décrite en termes variés au cours des années. Suivant une terminologie récente, la pile de saccules possède une face trans qui, dans les cellules glandulaires, donne naissance aux grains de sécrétion. Dans le même type de cellules, la face opposée à la face trans ou face cis est habituellement tournée vers les citernes du réticulum endoplasmique. La région du cytoplasme comprise entre la face cis de l'appareil de Golgi et les citernes du réticulum endoplasmique contient fréquemment des structures vésiculaires ou tubulaires groupées en amas, les éléments de transition, vraisemblablement responsables du transport des molécules entre le réticulum endoplasmique et l'appareil de Golgi (figure 2).

L'identification de la polarité de l'appareil de Golgi peut s'avérer difficile lorsque la cellule ne contient pas de grains de sécrétion, les marqueurs naturels de la face trans. On doit alors recourir à des techniques plus spécialisées qui mettent en évidence la polarité chimique de la pile de saccules. En effet, dès 1961, Alex Novikoff et ses collaborateurs [3] suggérèrent que certaines enzymes comme la thiamine pyrophosphatase

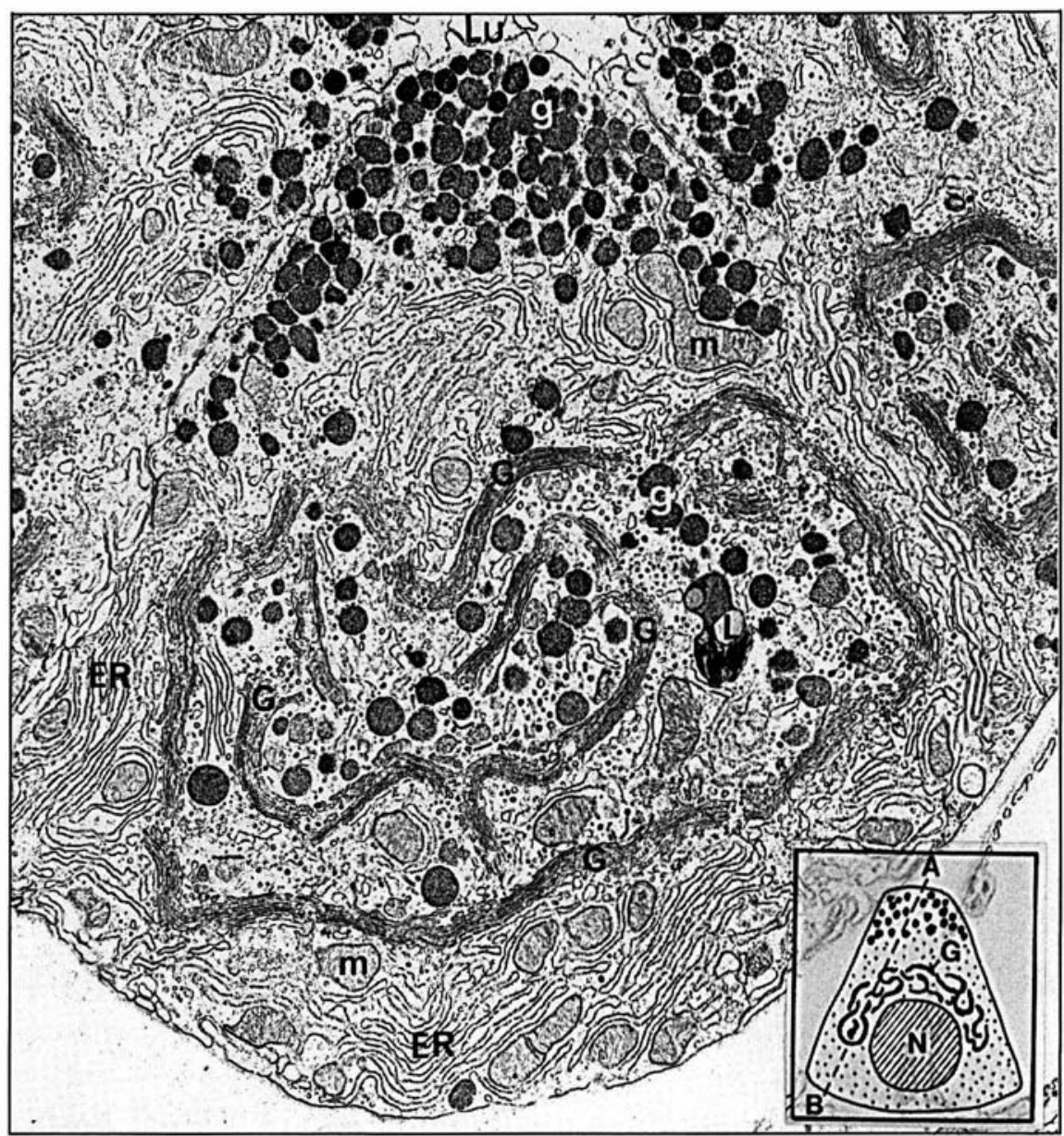

Figure 1. Image au faible grossissement d'une cellule à mucus de la glande de Brünner. Le noyau de la cellule n'est pas visible dans ce champ. Le plan de coupe de la cellule, indiqué par un dessin dans l'encart, passe par l'axe A-B. Le sommet et le pôle sécréteur de la cellule qui fait face à la lumière (Lu) contiennent de nombreux grains de sécrétion $(\mathrm{g})$. Dans le reste du cytoplasme, outre le réticulum endoplasmique (ER) et des mitochondries $(\mathrm{m})$, nous voyons le ruban contourné de l'appareil de Golgi (G) associé avec des grains de sécrétion en formation et des lysosomes (L). Ce ruban golgien qui apparaît discontinu sur coupe fine est en fait continu lorsque nous examinons des coupes plus épaisses. ( $\times 10500$.

tases (NDPases), ou la cytidine monophosphatase (CMPase) pouvaient être considérées comme des marqueurs de l'appareil de Golgi en microscopie optique. Les études ultérieures en microscopie électronique confirmèrent cette observation et montrèrent qu'effectivement la TPPase ou la CMPase étaient préférentiellement localisées dans les saccules situées du côté trans de la pile. Comme le notaient Friend et Murray en 1965 [4], les saccules de la face cis peuvent, dans certaines conditions, être sélectivement imprégnés par l'osmium. Plus récemment, Smith, en 1980, [5] a démontré que la détection de la nicotinamide adénine dinucléotide phosphatase (NADPase) pouvait être utilisée pour la mise en évidence des saccules intermédiaires ou éléments médians.

\section{Coupes épaisses et microscopie électronique tridimensionnelle}

Comme nous venons de le voir, les observations en microscopie électro- 


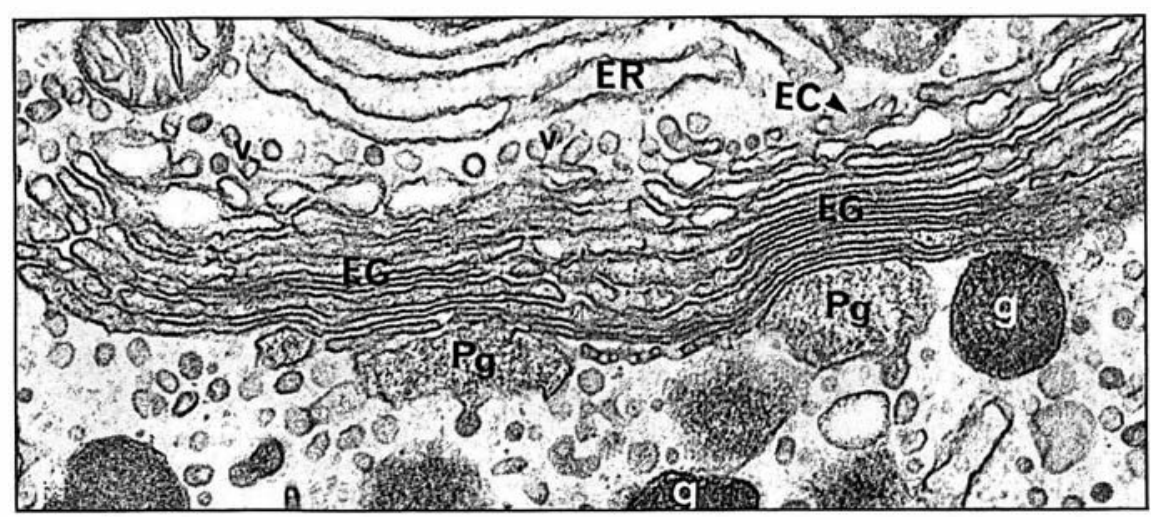

Figure 2. Coupe transversale du ruban golgien d'une cellule à mucus montrant la multiplicité et la variabilité structurale des éléments qui le composent. Dans le cas présent, l'axe cis-trans est indiqué par la présence de granules prosécrétoires (Pg) visibles sur la face trans. Le premier élément de la pile est l'élément cis (EC). Cet élément cis est séparé des citernes du réticulum endoplasmique par un espace contenant de petites vésicules de transfert (V). Les éléments golgiens sous-jacents à l'élément cis appartiennent aux compartiments médian et trans (EG). Certains éléments de ce dernier compartiment sont aplatis ; d'autres sont légèrement dilatés et contiennent un matériel granulaire qui se concentre dans les granules prosécrétoires. Des grains de sécrétion (g) se trouvent sur la face trans (voir figure 10 qui résume nos observations). ( $\times 50000$.)

nique sont effectuées sur coupes extrêmement fines et les informations recueillies par cette technique sont souvent trop fragmentaires lorsque la structure étudiée possède une architecture complexe et développée dans l'espace.

En 1969, l'un des auteurs (A. Rambourg [6]) étudiait la localisation de molécules riches en sucres (glycoprotéines) au niveau de l'appareil de Golgi des cellules intestinales. La technique utilisée ne faisait apparaître qu'une partie des structures cytoplasmiques, rendant ainsi les coupes plus transparentes aux électrons. En augmentant progressivement leur épaisseur, il constata qu'il était possible d'observer, à l'aide d'un microscope électronique conventionnel, des coupes dont l'épaisseur était dix à quinze fois supérieure à celle du matériel préparé par les méthodes habituelles. Les premières photographies prises dans ces conditions révélèrent immédiatement la complexité de la face trans de l'appareil de Golgi. A ce niveau, les structures sacculaires traditionnelles semblaient être interconnectées par des éléments tubulaires plus ou moins enchevêtrés.

$\mathrm{m} / \mathrm{s} n^{\circ} 1$ vol. 6, janvier 90 devait donc être neutralisé par l'utilisation de techniques stéréoscopiques.

Ces techniques permettent en effet d'observer, non la projection, mais la structure qui a donné naissance à cette projection. Elles sont fondées sur l'aptitude du cerveau à reconstruire un objet en trois dimensions à partir des projections, sur les deux rétines, d'un même objet vu par chaque œil sous un angle différent. En pratique, deux techniques stéréoscopiques sont couramment utilisées dans nos laboratoires. La première, relativement simple, consiste à prendre deux photographies d'une même structure en inclinant la platine

goniométrique (le porte-objet) du microscope électronique sous deux angles différents choisis en fonction de l'agrandissement et de l'épaisseur de la coupe. Ces deux photographies correspondent à deux projections différentes d'un même objet. La reconstitution tridimensionnelle de ce dernier pourra donc être obtenue en examinant les deux photographies placées côte à côte à l'aide d'un dispositif optique (stéréoscope ou projecteur stéréoscopique) permettant à chaque œil de voir exclusivement l'image qui lui est destinée. La seconde technique, plus sophistiquée, utilise les propriétés d'un système de lentilles cylindriques parallèles. Adaptée pour le microscope électronique, elle permet non seulement de voir directement l'objet en trois dimensions, mais aussi, comme sur un hologramme, d'examiner cet objet sous différentes incidences grâce à sa rotation dans l'espace.

Dans la majorité des cas, ces reconstitutions tridimensionnelles sont réalisées à partir d'un matériel examiné à l'aide du microscope électronique conventionnel qui accélère les électrons sous une tension maximale de 120000 volts. Lorsque l'épaisseur des coupes est trop importante, les prises de vue sont effectuées à l'aide d'un microscope électronique à très haute tension (un à trois millions de volts) qui, en raison de la forte accélération des électrons, donne des images satisfaisantes de coupes dont l'épaisseur peut atteindre dix à quinze micromètres.

\section{Architecture générale de l'appareil de Golgi}

Il y avait une contradiction apparente entre les données de la microscopie électronique et l'observation initiale de Golgi décrivant l'appareil réticulaire interne sous forme d'une structure continue. La découverte de Golgi avait été effectuée à la suite de l'imprégnation de cellules nerveuses par un dichromate d'osmium. Friend et Murray ont par ailleurs montré en 1965 [4] que l'imprégnation des tissus par l'osmium à chaud provoquait l'accumulation sélective de ce métal dans le saccule cis des piles de saccules observées sur coupes ultrafines en microscopie électroni- 


\section{RÉFÉRENCES}

1. Golgi C. Sur la structure des cellules nerveuses des ganglions spinaux. Arch Ital Biol $1898 ; 30: 278-86$

2. Dalton A J, Felix MP. Cytologic and cytochemical characteristics of the Golgi substance of epithelial cells of the epididymis-in situ, in homogenates and after isolation, $A m$ $J$ Anat 1954; 94 : 171-207.

3. Novikoff AP, Goldfischer S. Nucleoside diphosphatase activity in the Golgi apparatus and its usefulness for cytological studies. Proc Natl Acad Sci USA 1961 ; 47 : 802-10.

4. Friend DS, Murray M. Osmium impregnation of the Golgi apparatus, Am J Anat 1965 ; 117 : $135-50$.

5. Smith CF. Ultrastructural localization of nicotinamide adenine dinucleotide phosphatase (NADPase) activity in the intermediate saccules of the Golgi apparatus in rat incisor amioblasts. J Histochem Cytochem 1980 ; 28 : $16-26$.

6. Rambourg A. L'appareil de Golgi : examen en microscopie électronique de coupes épaisses $((, 5-l \mu)$ colorées par le mélange chlorhydrique-phosphotungstique. CR Acad Sci Paris 1969 ; D 269 : 2125-7.

7. Rambourg A, Clermont Y, Marraud A. Three-dimensional structure of the osmiumimpregnated Golgi apparatus as seen in the high voltage electron microscope. Am J Anat $1974 ; 140: 27-46$.

8. Rambourg A, Clermont $\mathrm{Y}$, Hermo L, Segrétain D. Tridimensional structure of the Golgi apparatus of non-ciliated epithelial cells of the ductuli efferentes in the rat: an electron microscope stereoscopic study. Biol Cell $1987 ; 60$ : 103-16.

9. Ramón y Cajal S. Algunas variaciones fisiologicas y patologicas del aparato reticular de Golgi. Trab Lab Invest Biol Univ Madrid 1914; 12 : 127-227.

10. Nassonov DN. Das Golgische Binnennetz und seine Beziehungen zu der Sekretion. Untersuchungen über einige Amphibiendrüsen. Arch Mikrosk Anat Entw Mech 1923 ; 97 : 136-86.

11. Bowen RH. The Golgi apparatus, it's structure and functional significance. Anat Rec 1926 ; 32 : 151-93.

12. Warshawsky $\mathrm{H}$, Leblond CP, Droz B. Synthesis and migration of proteins in the cells of the exocrine pancreas as revealed by specific activity determination from radioau- que. En utilisant cette technique et en augmentant progressivement l'épaisseur des coupes, on pouvait espérer connaître la structure tridimensionnelle de l'élément cis et, dans le même temps, établir une corrélation entre cet élément osmiophile et la structure observée par Golgi après imprégnation par le dichromate d'osmium. Des ganglions nerveux furent donc imprégnés par la méthode de Friend et Murray. Sur coupes ultrafines, les éléments osmiophiles correspondaient effectivement à la face cis des piles de saccules et étaient distribués sans connexion apparente dans le cytoplasme périnucléaire des cellules ganglionnaires (figure 3 ). En revanche, en observant des coupes d'épaisseur croissante, on mettait en évidence des structures osmiophiles de plus en plus longues et de plus en plus complexes qui, sur coupes très épaisses examinées en microscopie électronique à haute tension, étaient reliées les unes aux autres, formant le réseau périnucléaire décrit par Golgi dans ce type cellulaire (figures 4 et 5). Si, au cours d'autres expériences, on marquait sélectivement soit les éléments médians des piles de saccules par une technique de détection de la NADPase, soit l'élément trans grâce à son activité TPPasique, on retrouvait sur coupes épaisses des images similaires (figures 7 et 8 , p. 40). On confirmait ainsi, pour la première fois, la pertinence des observations de Golgi en démontrant sans ambiguïté que l'appareil de Golgi examiné sur coupes épaisses, en microscopie électronique, présentait une structure comparable à celle de l'appareil réticulaire interne observé en microscopie optique [7]. Comme l'avaient noté Golgi et ses successeurs immédiats, l'architecture générale de l'appareil de Golgi et son extension dans le cytoplasme varient considérablement d'un type cellulaire à l'autre. Dans les cellules ganglionnaires, le réseau intracytoplasmique formé par cet organite est particulièrement développé et peut s'étendre de la région nucléaire à la surface cellulaire (figure 5). Dans les autres types cellulaires, ses dimensions sont plus modestes et, dans la majorité des cas, l'appareil de Golgi a l'aspect d'un ruban enroulé sur luimême, présentant de nombreuses anastomoses et formant dans la région paranucléaire une masse aux contours variables (figures 7 et 8 ).

\section{Hétérogénéité ultrastructurale de l'appareil de Golgi}

Examiné à plus fort grossissement, le réseau intracytoplasmique imprégné par l'osmium présentait, dans les cellules ganglionnaires, une structure jusqu'alors totalement insoupçonnée puisque les travées de ce réseau étaient elles-mêmes constituées par un système de tubules étroitement

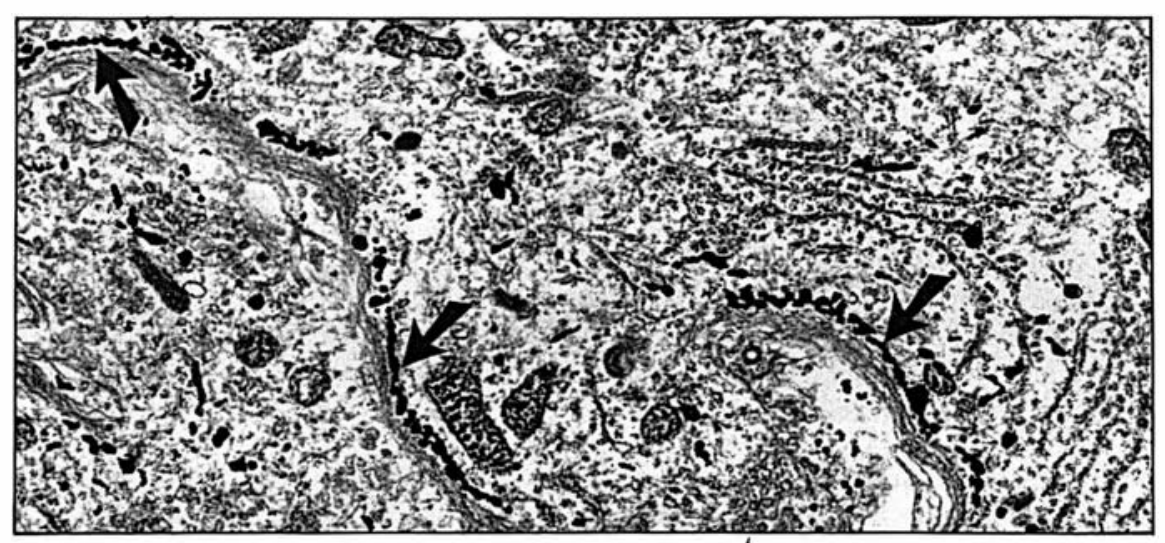

Figure 3. Coupe fine d'une cellule ganglionnaire colorée à chaud avec le tétroxyde d'osmium. L'osmium colore en noir l'élément cis du ruban golgien (flèches). Sur coupe fine, l'élément cis paraît discontinu. 


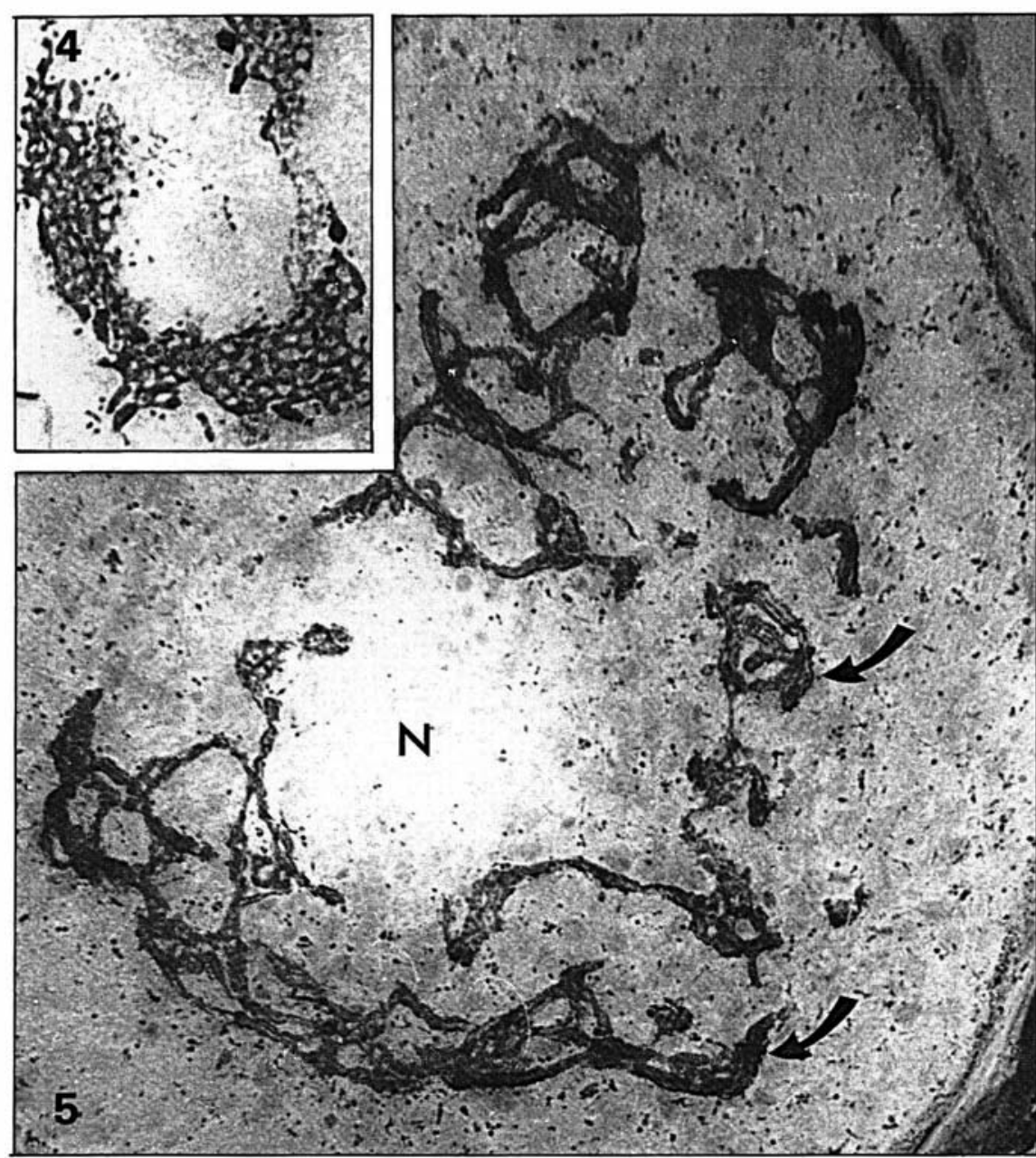

Figure 4 (en haut à gauche). Coupe de 1 um d'épaisseur d'une cellule nerveuse d'un ganglion spinal imprégnée par l'osmium et examinée avec un microscope électronique à haut voltage. L'élément osmiophile cis a l'aspect d'un réseau tubulaire à mailles serrées. Les autres éléments du ruban golgien ne se colorent pas par cette méthode. L'élément cis présente la même structure tubulaire tout le long du ruban golgien. ( $X 30000$.)

Figure 5. Coupe de 5 $\mathrm{mm}$ d'épaisseur d'une cellule nerveuse d'un ganglion spinal imprégnée par l'osmium et examinée avec un microscope électronique à haut voltage. Dans cette image au faible grossissement, l'élément osmiophile (flèches) s'étend autour du noyau (N), qui n'est pas coloré. II forme un large réseau dont de larges portions sont continues. Dans des coupes encore plus épaisses, le ruban golgien forme un réseau continu autour du noyau, comme l'avait déjà suggéré Golgi lui-même. (X 10000.)

anastomosés en un filet à petites mailles polygonales régulières (figure 4). Ainsi, contrairement à l'opinion communément admise, le réseau osmiophile correspondant au côté cis des piles de saccules ne contenait en réalité aucune portion sacculaire. En raison de sa structure particulière, retrouvée dans la plupart des types cellulaires examinés $\mathrm{m} / \mathrm{s} n^{\circ} 1$ vol. 6 , janvier 90 (figures 7 et 8). Cependant, en règle générale, le ruban continu formé par chacun de ces éléments était caractérisé par l'alternance de portions peu fenêtrées compactes, les portions sacculaires, et de portions plus lâches extrêmement perforées, voire exclusivement tubulaires, les portions intersacculaires.

Ces observations faisaient ressortir deux faits nouveaux : d'une part, une hétérogénéité de structure entre l'élément cis et les autres éléments de l'appareil de Golgi; d'autre part, une hétérogénéité longitudinale des éléments « médians » et trans.

Le problème se posait maintenant de la manière suivante: comment concilier ces données avec le modèle de la pile de saccules décrit sur coupes ultrafines? La solution évidente consistait à retourner à l'observation de coupes semi-fines en utilisant des méthodes d'imprégnation sélective des éléments golgiens.

\section{Compartiments dans I'axe cis-trans de l'appareil de Golgi}

L'analyse sur coupes fines de la structure tridimensionnelle de l'appareil de Golgi est particulièrement redoutable en raison des nombreuses torsions et replis du ruban golgien, qui peut ainsi être coupé sous n'importe quelle incidence. De ce fait, il est parfois difficile de reconnaître certaines structures et souvent malaisé de distinguer la face cis et la face trans. De plus la structure des divers éléments qui composent l'appareil de Golgi est très variable d'une région à l'autre du réseau golgien d'une même cellule. Enfin, l'organisation tridimensionnelle des éléments golgiens varie considérablement d'un type cellulaire à l'autre. La description qui va suivre, fondée sur des observations stéréoscopiques de l'appareil de Golgi des cellules épithéliales des cônes épididymaires [8], donne une image typique de l'appareil de Golgi des cellules ne produisant pas de grains de sécrétion (figure 2). Sur une coupe longitudinale et perpendiculaire à la surface du ruban golgien, on peut différencier trois compartiments qui se présentent de la manière suivante en commençant par la face cis.

(1) Le réseau tubulaire osmiophile 
Les figures 6, 7 et 8 sont des stéréopaires, c'est-à-dire des couples de deux photographies d'un même champ prises en inclinant le porte-objet du microscope électronique sous deux angles différents là savoir $\pm 9 \%$. L'examen de ces stéréopaires, à l'aide d'une lunette stéréoscopique bien ajustée à la distance de $65 \mathrm{~mm}$, permet à l'observateur d'avoir une image tridimensionnelle de l'objet photographié.
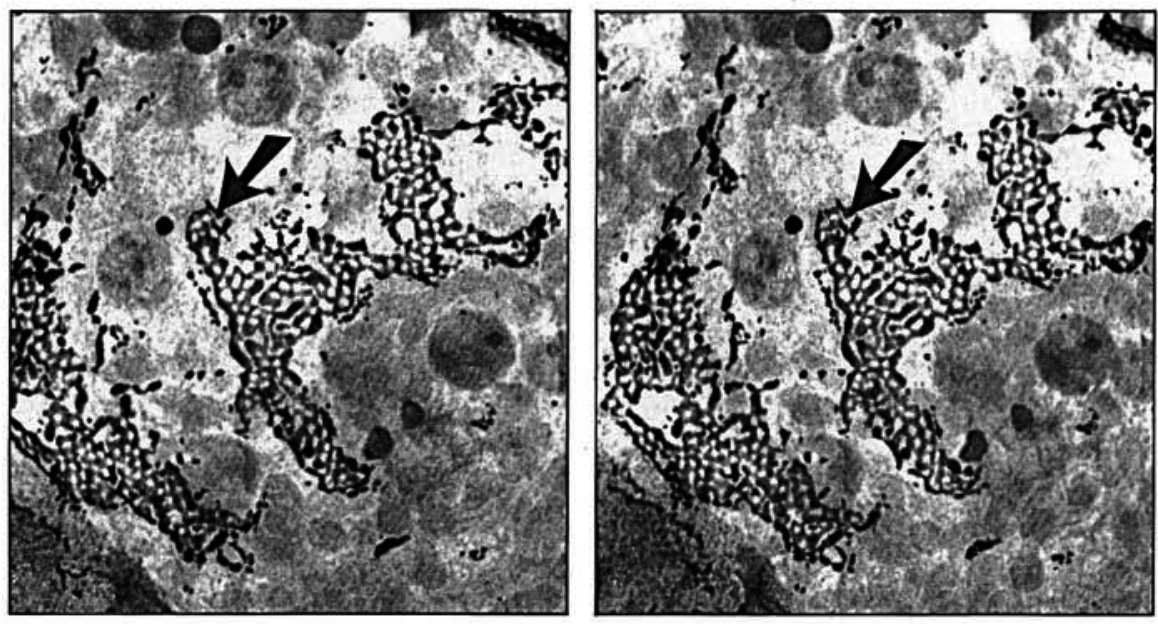

Figure 6. Image tridimensionnelle de l'élément osmiophile cis (flèche) d'une cellule épithéliale des cônes efférents. L'aspect tubulaire et en réseau de l'élément cis de même que sa continuité dans l'espace sont évidents quoique la coupe n'ait que $0,5 \mu m$ d'épaisseur. ( $\times 5$ 000.)
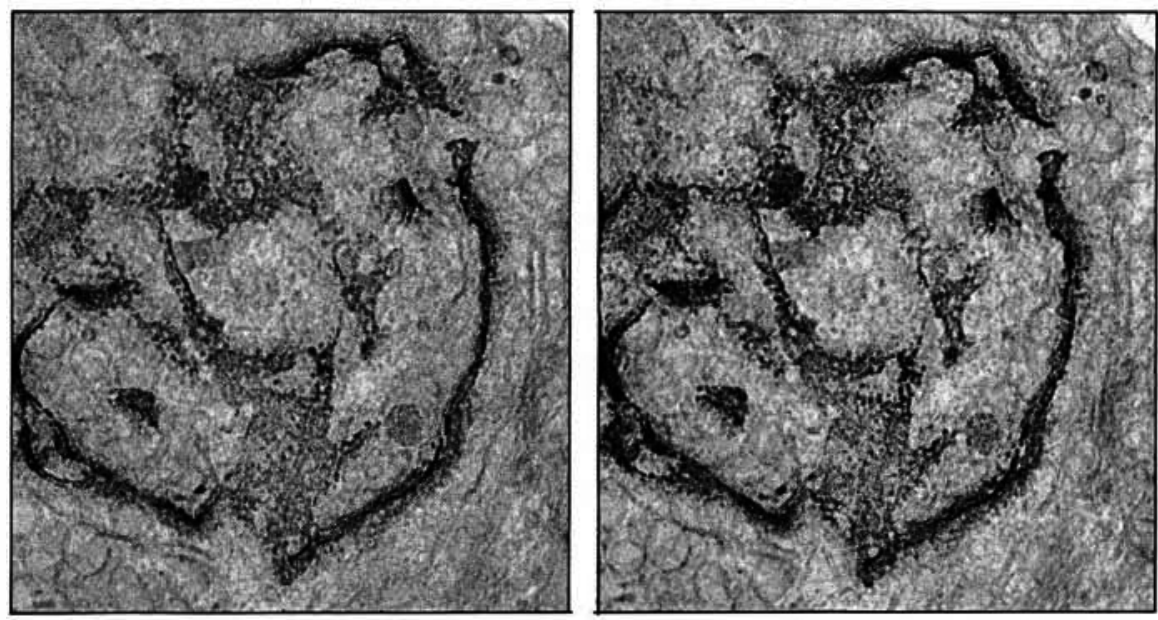

Figure 7. Image tridimensionnelle d'un appareil de Golgi d'une celIule à prolactine de I'hypophyse de rat. Dans cette coupe de $3 \mu \mathrm{m}$ d'épaisseur un ou deux éléments médians du ruban golgien ont été colorés par une méthode révélant une activité phosphatasique (NADPase). II apparaît clairement que l'appareil de Golgi est composé d'un ruban continu présentant de nombreuses circonvolutions et anastomoses. (X 6 000.)
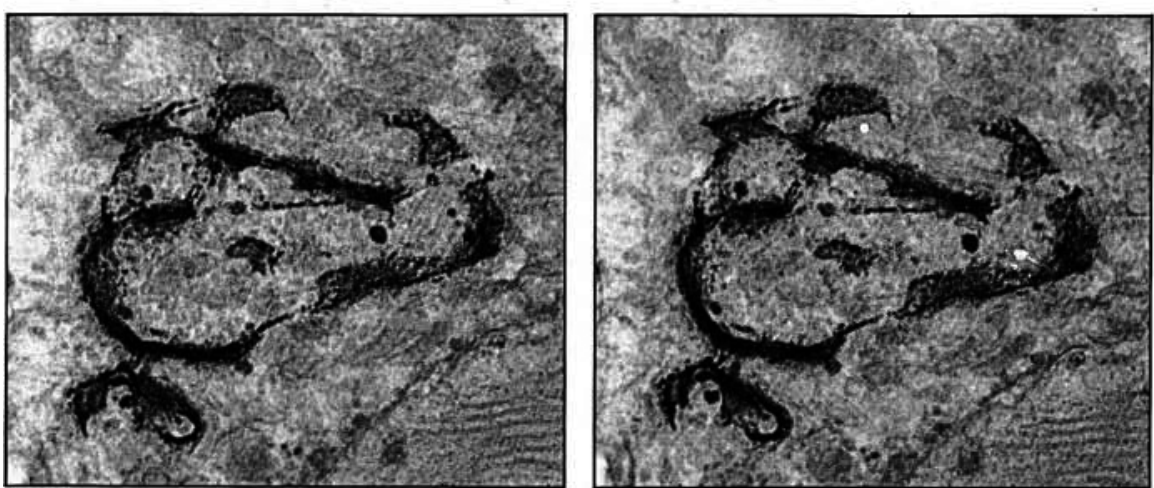

Figure 8. Image tridimensionnelle de l'appareil de Golgi d'une cellule à prolactine de l'hy pophyse de rat. Dans cette coupe de $3 \mu \mathrm{m}$ d'épaisseur. un ou deux éléments porteurs d'activité TPPase du compartiment trans ont été colorés d'une façon sélective. II apparaît que dans ce cas-ci également, le où les éléments TPPase positifs forment une structure continue avec des circonvolutions et anastomoses. ( $\times 6000)$. 
ou compartiment cis, a l'apparence d'une rangée de profils vésiculaires reliés les uns aux autres par des ponts de densité variable.

(2) Immédiatement sous l'élément osmiophile cis, trois ou quatre éléments sacculo-tubulaires disposés de manière strictement parallèle forment ce que nous définirons comme le compartiment «médian». La superposition de leurs portions sacculaires se reconnaît aisément et correspond de toute évidence aux piles de saccules traditionnellement décrites. Les portions sacculaires des deux ou trois éléments situés sous le réseau tubulaire cis contiennent parfois la NADPase [8]. Elles sont le siège de larges perforations localisées dans des régions homologues des saccules superposés. Alignées suivant l'axe cis-trans, elles forment dans l'espace des puits évasés dont l'ouverture est toujours orientée vers la face cis du ruban golgien et dont le fond est toujours constitué par la portion sac- culaire de l'élément sous-jacent riche en TPPase [8]. Ces puits contiennent des petites vésicules dont le diamètre est de 80 nanomètres.

(3) Le compartiment trans est constitué de plusieurs éléments sacculo-tubulaires dont la configuration spatiale variable diffère notablement de celle des éléments susjacents. Ces éléments présentent une portion sacculaire peu fenêtrée, étroitement accolée et strictement parallèle à la portion sacculaire des élé-

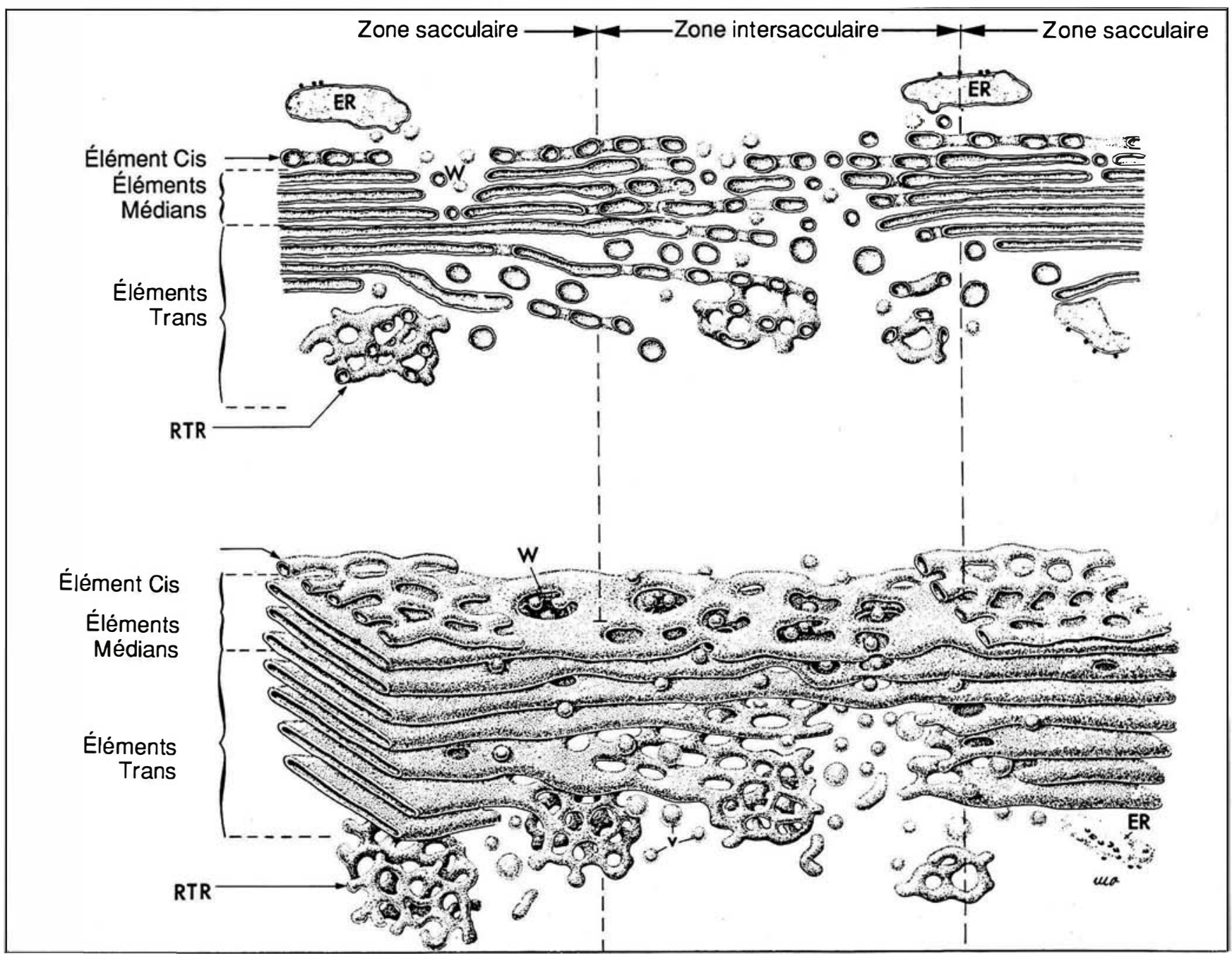

Figure 9. Diagramme montrant une petite portion du ruban golgien d'une cellule non sécrétrice (cellule épithéliale des cônes efférents du rat). Le dessin du haut montre l'empilement des éléments golgiens tel qu'on les voit dans une coupe transversale réalisée dans l'axe du ruban. Le dessin du bas montre une image tridimensionnelle des divers éléments du ruban golgien reconstitués par nos images stéréoscopiques. L'élément cis est un élément continu mais, dans ce dernier dessin, il est interrompu pour permettre la représentation des éléments sous-jacents. Le ruban golgien montre des zones compactes ou sacculaires et des zones fenestrées et tubulaires ou intersacculaires. Tandis que les éléments du compartiment médian sont continus le long du ruban, les éléments du compartiment trans sont souvent discontinus et semblent se détacher des éléments sus-jacents. Dans les zones compactes, on note la présence de puits (W) contenant de petites vésicules et sur la face trans, on remarque la présence de réseaux tubulaires résiduels (RTR) (voir la description détaillée dans le texte). 


\section{RÉFÉRENCES}

13. Palade G. Intracellular aspects of the process of protein secretion, Science 1975 189 : 347-58.

14. Rambourg A, Clermont Y, Hermo L Segrétain D. Tridimensional architecture of the Golgi apparatus and its components in mucous cells of Brunner's gland of the mouse, Am J Anat 1987; 179 : 95-107.

15. Hand AR, Oliver C. Relationship between the Golgi apparatus, GERL, and secretory granules in acinar cells of the rat exorbital lacrimal gland. J Cell Biol 1977; 74 : 399-413.

16. Von Zastrow M, Castle JD. Protein sorting among two distinct export pathways occurs from the content of maturing exocrine storage granules. J Cell Biol 1987 ; 105 : 2675 . 84 .

17. Rambourg A, Clermont Y, Hermo L Formation of secretion granules in the Golgi apparatus of pancreatic acinar cells of the rat. Am J Anat 1988; 183 : 187-99.

18. Rambourg A, Clermont $\mathrm{Y}$, Hermo L Chrétien M. Formation of secretion granules in the Golgi apparatus of plasma cells of the rat. Am J Anat 1989 ; 183 : 187-99.

19. Leblond CP. Distribution of periodic acid-reactive carbohydrates in the adult rat. Am J Anat 1950 ; 86 : 1-49.

20. Thiery JP. Rôle de l'appareil de Golgi dans la synthèse des mucopolysaccharides. Étude cytochimique. I. Mise en évidence de mucopolysaccharides dans des vésicules de transition entre l'ergastoplasme et l'appareil de Golgi. J Microscopie 1969 ; 8 : 689-708.

21. Rambourg A, Hernandez W, Leblond CP Detection of complex carbohydrates in the Golgi apparatus of rat cells. J Cell Biol 1969 40 : $395-414$.

22. Neutra M, Leblond CP. Synthesis of the carbohydrate of mucus in the Golgi complex as shown by electron microscope radiography of goblet cells from rats injected with glucose$\mathrm{H}_{3}$. J Cell Biol 1966; 30 : 119-36.

23. Leblond CP, Bennett G. Role of the Golg apparatus in terminal glycosylation. In Brinkley BR, Porter K, eds. International cell biology. New York: Rockefeller University Press, 1977 : 326-36.

24. Roth J, Taatjes DJ, Lucocq JM, Weinstein J, Paulson JC. Demonstration of an extensive trans-tubular network continuous with the Golgi apparatus stack may function in glycosylation. Cell 1985 ; 43 : 287-95.

25. Kornfeld R, Kornfeld S. Assembly of asparagine-linked oligosaccharides. Annu Rev Biochem 1985; 54 : 631-64. ments sus-jacents. Elle est en continuité à sa périphérie et dans toutes les directions de l'espace avec une portion tubulaire qui s'écarte du ruban golgien en s'incurvant dans le cytoplasme de la région trans (figure 9, p. 41). Les éléments anastomosés de cette portion tubulaire forment un réseau à mailles polygonales plus larges que celles du réseau tubulaire cis. Le premier de ces éléments est souvent porteur d'une activité TPPase, alors que les autres contiennent souvent la CMPase. Outre les éléments sacculo-tubulaires, on rencontre encore, dans la zone trans de l'appareil de Golgi, de nombreuses vésicules ou tubules membranaires ainsi que des portions terminales de citernes du réticulum endoplasmique, étroitement accolées aux éléments sacculo-tubulaires.

\section{Fonctions}

\section{de l'appareil de Golgi}

- Formation des grains de sécrétion. L'implication de l'appareil de Golgi dans la genèse des produits de sécrétion a été reconnue dès 1914 par le pionnier de la cytologie du système nerveux, le biologiste espagnol Santiago Ramón y Cajal [9]. Examinant au microscope optique les cellules à mucus caliciformes chargées, dans l'intestin, de déverser une couche de mucus protecteur à la surface de la muqueuse, il remarqua la présence de fines gouttelettes de mucus dans la région de l'appareil de Golgi. Des observations similaires ont été rapportées par Nassonov en 1922 [10] dans les cellules pancréatiques. En 1929 Bowen [11] décrivait la formation, dans l'appareil de Golgi, de l'acrosome : un lysosome volumineux localisé à la surface du noyau des spermatides, les cellules germinales à l'origine des spermatozoïdes. Il fallut néanmoins attendre l'utilisation des isotopes radioactifs en biologie pour apporter la preuve définitive du rôle joué par l'appareil de Golgi dans la synthèse, la modification et la concentration des produits de sécrétion dans les cellules glandulaires.

La autoradiographie, une technique mise au point par Charles Leblond et ses collaborateurs à l'université McGill de Montréal, allait permettre de visualiser, en 1963, la concentration d'un produit de sécrétion dans la région golgienne des cellules du pancréas exocrine [12]. Des acides aminés marqués radioactivement étaient injectés à des rats et à des souris. Après avoir mesuré la radioactivité et la concentration relative en protéines des divers organites, Warshawsky, Droz et Leblond [12] aboutirent à la conclusion que les protéines synthétisées dans le réticulum endoplasmique se déplaçaient vers la région golgienne pour y être concentrées et empaquetées dans les grains de sécrétion contenant les enzymes du suc pancréatique.

Les études ultérieures ont combiné des techniques de autoradiographie en microscopie électronique à l'analyse biochimique de fractions cellulaires après injection d'isotopes radioactifs. Elles ont permis de préciser les différentes étapes du processus sécrétoire. On a pu ainsi montrer, à la suite notamment des travaux de G. Palade et al. [13], que, dans de nombreuses cellules glandulaires, le matériel sécrétoire s'accumulait et se concentrait dans les extrémités latérales dilatées des saccules trans des piles de saccules observées sur coupes ultrafines.

Après avoir démontré la continuité et la complexité de l'appareil de Golgi, il nous fallait expliquer comment pouvaient s'effectuer, dans une telle structure, la ségrégation du matériel sécrétoire et son empaquetage dans les grains de sécrétion. Un premier élément de réponse a été obtenu en 1987 en examinant sur coupes épaisses, en trois dimensions, l'ultrastructure de l'appareil de Golgi dans une cellule dont le produit de sécrétion était spontanément opaque aux électrons : la cellule à mucus de la glande de Brünner localisée dans la paroi de l'intestin grêle [14].

Dans ce type cellulaire, l'appareil de Golgi se présente sous la forme d'un long ruban replié sur lui-même et localisé dans la région supranucléaire, entre le noyau et la masse des grains de sécrétion groupés au pôle sécrétoire de la cellule (figure 1). Sur une coupe longitudinale et perpendiculaire à la surface du ruban, on reconnaît les trois compartiments que nous avons identifiés dans les autres types cellulaires, c'est-à-dire le réseau tubulaire cis, le compartiment «médian » et le compartiment trans (figures 2 et 10). 


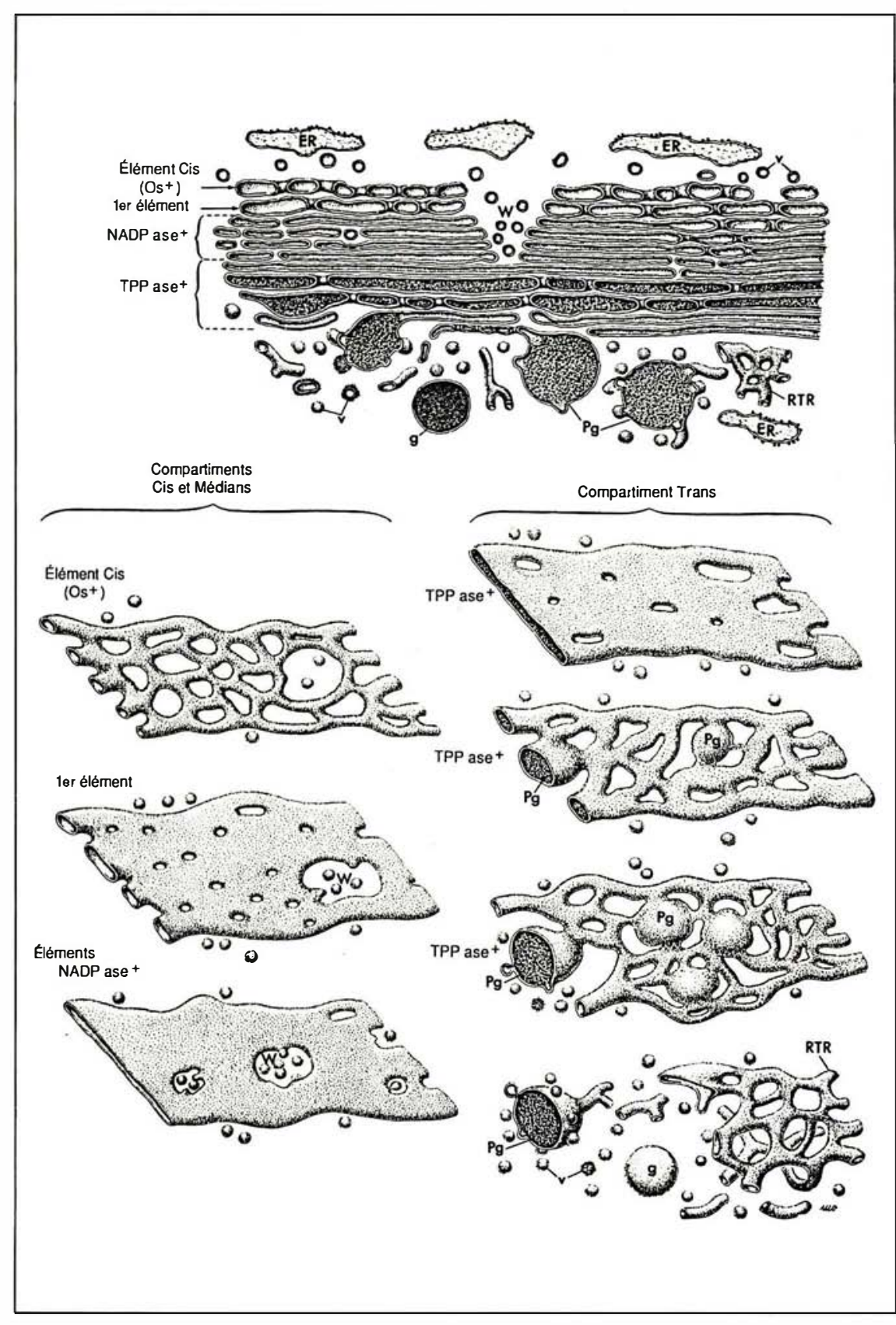

Figure 10. Diagramme montrant unetrès petite portion du ruban golgien d'une cellule à mucus d'une glande de Brünner du duodénum de souris. Le dessin du haut montre une coupe transversale du ruban golgien, les divers éléments qui le composent, ainsi que l'imprégnation par le tétroxyde d'osmium (Os), la présence d'une activité nicotine amide dinucléotide phosphatase (NADPase) ou thiamine pyrophosphatase (TPPase). Dans le dessin du bas, ces mêmes éléments sont illustrés isolément et en vues tridimensionnelles. A gauche, l'élément cis osmiophile a l'aspect d'un réseau tubulaire. Le $1^{\text {er }}$ élément du compartiment médian sous-jacent montre de petits pores mais n'est pas NADPase positif. Les trois éléments suivants du compartiment médian NADPase positif sont illustrés par un seul dessin. Ces éléments comme le 1er élément sont perforés par de grands trous alignés qui forment des puits (W) contenant des petites vésicules. A droite, les divers éléments du compartiment trans TPPase positif et contenant du matériel sécrétoire sont représentés en trois dimensions. Ces dessins illustrent le mode de formation probable des grains de sécrétion (voir détail dans le texte).

$\mathrm{m} / \mathrm{s} n^{\circ} 1$ vol. 6 , janvier 90
Le matériel sécrétoire apparaît dans le premier élément porteur d'une activité TPPase sous la forme d'une substance finement granuleuse répartie uniformément dans la lumière du saccule. Au niveau des deux éléments suivants, on observe une fenestration des saccules, tandis que le matériel sécrétoire s'accumule dans des dilatations localisées au hasard et non exclusivement sur les bords des saccules, comme le suggéraient les théories classiques [15]. Au niveau du dernier élément trans, les dilatations volumineuses contiennent un matériel dense aux électrons et sont interconnectées par des portions sacculaires aplaties très fenêtrées et par des portions tubulaires complètement dépourvues de matériel sécrétoire. Dans le cytoplasme séparant les éléments trans, de volumineux granules contiennent un matériel de densité analogue à celui des dilatations sacculaires. Ce sont les granules prosécrétoires - dont la surface, irrégulière, est souvent hérissée de résidus tubulaires. Certains granules sont entourés d'une couronne de petites vésicules. D'autres granulations, sphériques et moins volumineuses, correspondent aux grains de sécrétion. Elles renferment un matériel très dense aux électrons et possèdent une surface parfaitement lisse (figure 2 et 10).

Les images observées dans les cellules de la glande de Brünner suggèrent fortement que le matériel sécrétoire, d'abord distribué de manière homogène dans les éléments sacculaires, s'accumule ensuite en des régions localisées au hasard pour former des dilatations qui donneront naissance aux granules prosécrétoires. Le drainage du matériel vers ces dilatations s'accompagne d'une perforation progressive de larges portions sacculaires qui se vident de leur contenu, se fragmentent et permettent ainsi la libération des granules prosécrétoires. La transformation des granules prosécrétoires en grains de sécrétion s'effectuerait grâce à l'émission de petites vésicules qui provoquerait une réduction du volume des granules et la concentration progressive de leur contenu. Une telle hypothèse a déjà été proposée $\$ par von Zastrow et Castle [16] dans une étude de la formation des grains de sécrétion par les cellules de la parotide du 
rat. Comme nous l'avons montré récemment, les grains de sécrétion se formeraient d'une manière analogue dans les cellules du pancréas exocrine [17] et le plasmocyte [18].

- Glycosylation des protéines. Le rôle joué par l'appareil de Golgi dans la modification des protéines après leur synthèse dans le réticulum endoplasmique (modifications posttraductionnelles) a été pressenti dès 1950 lorsque Leblond [19] montra en microscopie optique que l'acide périodique Schiff, qui permet de détecter les sucres, colorait l'appareil de Golgi de nombreux types cellulaires. Dès que des techniques similaires purent être utilisées en microscopie électronique, Jean-Paul Thiery en France [20], Rambourg, Hernandez et Leblond[21] au Canada montrèrent que les piles de saccules étaient marquées par ce réactif et que l'intensité de la réaction augmentait de la face cis vers la face trans de ces organites. Ces observations suggéraient que des molécules de sucres étaient progressivement ajoutées aux protéines lors de leur passage dans l'appareil de Golgi. De fait, lorsqu'en 1966 Neutra et Leblond [12] examinèrent par la technique autoradiographique les tissus d'animaux ayant reçu du galactose tritié dans les minutes précédant leur sacrifice, les grains d'argent étaient localisés dans la région golgienne de nombreux types cellulaires.

Dans les années qui suivirent, Leblond et al. [23] étudièrent par autoradiographie l'incorporation de différents sucres dans une large variété de types cellulaires. Ils purent ainsi établir que les sucres localisés à l'extrémité de chaînes polysaccharidiques branchées sur les protéines (glycoprotéines) étaient incorporés dans l'appareil de Golgi. En revanche, les sucres situés au voisinage de l'axe protéique des glycoprotéines étaient incorporés dans le réticulum endoplasmique.

Depuis cette époque, de nombreux travaux ont analysé et démontré la complexité des mécanismes qui régissent la synthèse des glycoprotéines au niveau du réticulum endoplasmique et de l'appareil de Golgi. Des enzymes catalysant l'addition ou la soustraction de sucres au cours de

préparation d'anticorps contre ces molécules a rendu possible leur localisation par immunocytochimie en microscopie électronique. Ainsi par exemple, dès 1982, Jürgen Roth et al. [24] localisaient dans le saccule riche en TPPase la galactosyltransférase permettant l'accrochage du galactose au voisinage de l'extrémité des chaînes polysaccharidiques. En revanche, la sialyltranférase, catalysant l'addition d'un sucre terminal : l'acide sialique, était détectée dans les éléments du réseau sacculo-tubulaire trans, riches en phosphatase acide.

- Ségrégation de molécules. De nombreux produits de sécrétion, les enzymes présentes dans les lysosomes et certains constituants de la membrane cytoplasmique sont des glycoprotéines et doivent par conséquent achever leur synthèse dans l'appareil de Golgi. La question s'est donc rapidement posée de savoir comment ces diverses macromolécules pouvaient être triées et dirigées vers leurs destinations respectives. A l'heure actuelle, seuls les mécanismes relatifs au tri des enzymes lysosomiales ont commencé à être élucidés. Kornfeld et Kornfeld [25] ont en effet montré qu'un ou plusieurs mannoses de ces glycoprotéines étaient porteurs d'un groupement phosphate, vraisemblablement ajouté à la molécule lors de son passage dans la région cis de l'appareil de Golgi. Le tri des enzymes se ferait ensuite par interaction des résidus mannose-phosphate avec un récepteur spécifique situé dans la membrane d'un ou plusieurs compartiments golgiens. On sait par ailleurs que certains récepteurs de la membrane cytoplasmique, chargés d'incorporer dans le cytoplasme des molécules d'origine exogène, sont périodiquement recyclés au niveau de l'appareil de Golgi. Le ou les compartiments impliqués dans ces phénomènes n'ont pas encore été identifiés avec certitude.

\section{Conclusion}

Les études tridimentionnelles réalisées dans nos laboratoires depuis une quinzaine d'années ont mis en évidence la complexité ultrastructurale de l'appareil de Golgi. Comme l'avait soupçonné Golgi, il s'agit d'un organite continu dont l'extension et l'organisation dans l'espace sont caractéristiques d'un type cellulaire donné et, vraisemblablement, de sa situation fonctionnelle. Dans un grand nombre de types cellulaires, il se présente sous la forme d'un long ruban replié sur lui-même, constitué de plusieurs éléments dont la structure semble déterminée par leur position suivant un axe cis-trans. A l'exception du réseau tubulaire cis, seul élément purement tubulaire, les autres éléments du ruban golgien présentent généralement une alternance de portions sacculaires et de portions lâches, extrêmement fenêtrées, voire exclusivement tubulaires. Situés immédiatement sous le réseau tubulaire osmiophile qui représente le compartiment cis, plusieurs éléments, étroitement superposés et strictement parallèles forment un compartiment «médian » dont les portions sacculaires sont sélectivement perforées par des puits. Le fond des puits est toujours constitué par un élément ayant une activité TPPase, qui pourrait représenter une structure de transition entre le compartiment «médian » et le compartiment trans. Les éléments sacculotubulaires trans sont séparés les uns des autres par des zones de cytoplasme renfermant diverses structures de nature vésiculaire ou tubulaire. Anastomosés au niveau de leurs portions tubulaires périphériques, ils forment le long du ruban golgien un ou plusieurs réseaux sacculo-tubulaire trans.

L'analyse tridimensionnelle de l'appareil de Golgi de plusieurs cellules glandulaires a souligné l'importance du rôle joué par le compartiment trans dans l'empaquetage, la libération et la concentration de certains produits de sécrétion. Les images observées suggèrent que la libération des granules prosécrétoires s'effectue par fenestration progressive et rupture des éléments trans. Le même compartiment et des mécanismes similaires sont vraisemblablement à l'origine de l'empaquetage et de la libération des glycoprotéines destinées aux lysosomes et à la surface cellulaire. Cependant, les structures responsables du transport de ces molécules n'ont pas encore été identifiées avec certitude et l'adéquation entre la structure des divers éléments golgiens et leurs fonctions est encore loin d'être réalisée 


\section{Summary}

The Golgi apparatus : contribution of tridimensional electron microscopy to the study of its structure and functions.

Our tridimensional studies on thin and thick sections of cells with the electron microscope have emphasized the ultrastructural complexity of the Golgi apparatus. As initially proposed by Golgi himsel, this organelle takes the appearance of a continuous and branching ribbon-like structure the overall configuration of which vary significantly from one cell type to another. In contrast to the cis-osmiophilic tubular network, the only purely tubular element of the Golgi apparatus, the other elements composing the ribbon usually display along their length, saccular zones and highly fenestrated or tubular intersaccular zones. These saccular and fenestrated zones are in register thus forming along the ribbon alternating compact and non compact zones. Subjacent to the cis-element making up the ciscompartment, one to four elements closely superposed and parallel to each other form the mid-compartment, the saccular zone of which are selectively perforated by wells. A third compartment or trans compartment consists of several sacculotubular elements which, in contrast to the mid-elements do not exactly follow the main axis of the Golgi ribbon and thus show a peelingoff configuration. The three dimensional analysis of the Golgi apparatus in glandular cells underlines the role of the trans compartment in the final segregation of secretion granules. The observations strongly suggest that the formation and liberation of prosecretory granules are concomitant with, and possibly result from a progressive fenestration and rupture of the trans elements.

\section{TIRÉS A PART}

Y. Clermont.

$\mathrm{m} / \mathrm{s} n^{\circ} 1$ vol. 6 , janvier 90
FLASH

Un article de l'équipe suédobritannique regroupée autour d'Olle Lindvall et d'Anders Björklund (à paraître dans la revue Science en janvier 1990) présente les résultats positifs d'une transplantation de neurones fœtaux dopaminergiques chez un malade parkinsonien. Le développement du greffon dans le putamen gauche du patient a été suivi à l'aide d'un marquage par fluoro-Dopa en caméra à positons. L'effet clinique, apparu deux mois après transplantation, est majeur sur la durée et la sévérité des périodes off qui passent de $50 \%$ à moins de $20 \% d u$ temps éveillé. Alors qu'il s'agit d'une greffe unilatérale, qui améliore comme on $s^{\prime} y$ attendait les mouvements alternatifs du côté opposé du corps, la rigidité est, pour sa part, nettement diminuée bilatéralement, ce qui est une relative surprise. II s'agit de la première publication présentant en parallèle des preuves de la survie d'un greffon neuronal chez l'homme et celles d'une amélioration clinique majeure. Ces résultats justifient ainsi les espoirs qu'avaient soulevées les données acquises depuis une dizaine d'années grâce aux greffes neuronales réalisées dans des modèles expérimentaux de la maladie de Parkinson.

Marc Peschanski

\section{$\mathbf{M S}$ à paraitre}

février 1990

VIROLOGIE

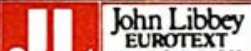

EUROTIEXT 